\title{
Effect of administration of phenylephrine immediately after low dose insemination on pregnancy rates in mares
}

\author{
Theresa Beachler ${ }^{1}$, Renan Sper ${ }^{1}$, C. Scott Bailey ${ }^{1 \#}$, James Shewmaker², C. Nick Buchanan, \\ Anne Josson-Schramme ${ }^{1,3}$, Michael Whitacre ${ }^{1}$ \\ ${ }^{1}$ College of Veterinary Medicine, North Carolina State University, Raleigh, USA \\ ${ }^{2}$ School of Veterinary Medicine, Ross University, Basseterre, Saint Kitts and Nevis \\ ${ }^{3}$ Current Address: VetAgro Sup, Université de Lyon, Lyon, France \\ Email: ${ }^{\text {scott_bailey@ncsu.edu }}$
}

Received 17 October 2013; revised 15 November 2013; accepted 23 November 2013

Copyright (C) 2013 Theresa Beachler et al. This is an open access article distributed under the Creative Commons Attribution License, which permits unrestricted use, distribution, and reproduction in any medium, provided the original work is properly cited. In accordance of the Creative Commons Attribution License all Copyrights (c) 2013 are reserved for SCIRP and the owner of the intellectual property Theresa Beachler et al. All Copyright (C) 2013 are guarded by law and by SCIRP as a guardian

\begin{abstract}
There is considerable pressure on equine veterinarians to achieve good pregnancy rates with very small doses of semen. Phenylephrine administration in the rabbit increased numbers of oviductal, uterine, and cervical sperm, myometrial contractions, and fertilized ova after low dose insemination. The use of phenylephrine to enhance uterine contractility and fertility has not been investigated in the mare. Thus, the objective of this study was to determine if phenylephrine administration would result in clinically acceptable pregnancy rates in mares bred by low dose insemination. The hypothesis (Ha) was that pregnancy rates would be significantly higher in mares receiving phenylephrine compared to saline controls. Six pony mares and eight horse mares were enrolled in this study. Mares were inseminated within 24 hours of ovulation with $\mathbf{3 0}$ million progressively motile spermatozoa from a single fertile stallion. Immediately following insemination, mares were administered either phenylephrine $(0.06 \mathrm{mg} / \mathrm{kg})$ or $1 \mathrm{~mL}$ of saline via IV injection. Pregnancy status was determined 14 days following ovulation via transrectal ultrasonography. Pregnancy rates in phenylephrine treated mares were $44 \%(4 / 9)$ while $22 \%(2 / 9)$ in saline-treated mares $(P>0.05)$.
\end{abstract}

Keywords: Artificial Insemination; Low Dose Insemination; Equine; Phenylephrine

*CONFLICT OF INTEREST: None of the authors has any financial or personal relationships that could inappropriately influence or bias the content of the paper.

\#Corresponding author.

\section{INTRODUCTION}

Low dose insemination provides the opportunity to increase the number of offspring a valuable stallion can produce in a season, and extends the availability of frozen semen from deceased or infertile stallions. In addition, the development of new advanced reproductive techniques, such as gender selection of equine sperm and the use of density gradients for centrifugation, places pressure on the equine industry to achieve acceptable pregnancy rates with very low numbers of sperm. It is known from work in horses as well as other species that only a fraction of the original inseminate arrives in the oviducts following natural service or standard artificial insemination [1-3]. Therefore, techniques that maximize the percentage of sperm that reach the oviduct may improve pregnancy rates when fewer sperm are used. Techniques that have been previously described in the mare to enhance sperm delivery to the oviduct include rectally guided deep horn insemination and hysteroscopic insemination [4-7]. As both of these are technically demanding and not widely available, there is a need for alternate methods of reducing the total number of sperm in a breeding dose while maintaining acceptable per cycle pregnancy rates. One such method would be the pharmacologic stimulation of sperm transport through enhancement of uterine contractility at the time of breeding.

In the mare, the exogenous administration of both prostaglandin E2 ( $\mathrm{PGE}_{2}$ ) and oxytocin has been investigated to facilitate gamete transport with disappointing or inconsistent results [8,9]. In contrast, administration of alpha adrenergic agonists has resulted in increased fertility after low dose insemination in the rabbit and ewe [10-12]. 
Phenylephrine, an alpha adrenergic agonist, is relatively selective for $\alpha 1$ receptors over $\alpha 2$ receptors. It is commonly used in animals as a decongestant, mydriatic agent, and to diagnose Horner's Syndrome. The primary effect of activation of $\alpha 1$ adrenoceptors is to increase smooth muscle tone, corresponding to an increase in intracellular calcium. In rabbit does, phenylephrine has been shown to increase frequency and amplitude of primary uterine contractions and the frequency of secondary contractions [10]. The administration of phenylephrine at the time of breeding resulted in the recovery of increased numbers of spermatozoa from the cervices, uterus and oviducts of sacrificed does [10]. The same authors demonstrated that phenylephrine administration increased the frequency of uterine contractions in ewes, but did not find improved sperm recovery from the oviducts of sacrificed animals [11,12]. Fertility trials have not been performed in the ewe, but administration of phenylephrine at time of low-dose insemination has been shown to increase recovery of fertilized ova from rabbit does [10]. No previous work has been conducted investigating this aspect of phenylephrine administration in the mare.

Therefore, the objective of this study was to determine whether administration of phenylephrine immediately after insemination would result in clinically acceptable pregnancy rates in mares that had been inseminated with low numbers of sperm. We hypothesized that pregnancy rates would be significantly higher in mares that received phenylephrine compared to those that received a saline control.

\section{MATERIALS AND METHODS}

Six pony mares and eight Quarter Horse or Thoroughbred mares, as well as one Quarter Horse stallion, were used in this study during the months of June through August. Mares were three to 15 years of age and were housed on pasture at two university-owned farms in central North Carolina. Seven mares were housed at the Teaching Animal Unit in Raleigh NC, and seven mares were housed at the Equine Health Center at Southern Pines. The stallion was maintained in an individual pasture on the grounds of the Equine Health Center at Southern Pines. All procedures were in accordance with North Carolina State University's Institutional Animal Care and Use Committee's guidelines for the humane treatment of research animals (IACUC ID\#10-048-O). All animals had a complete breeding soundness exam prior to enrollment in the study, including ultrasound exam of the uterus, speculum and digital exam of the vagina and cervix and a uterine biopsy. Mares were cycling regularly and had no evidence of reproductive complications on examination. At the onset of the study, mares were randomly divided into two separate groups, one receiving phenylephrine and one receiving saline. A cross-over study design was used in which treatment groups were switched for subsequent estrous cycles, resulting in a total of 28 cycles in the study. Transrectal palpation and ultrasonographic examinations of the reproductive tract were performed every other day for breeding management. Mares identified in early to mid diestrus were given Prostaglandin $\mathrm{F}_{2 \alpha}$ (Lutalyse, Pfizer, 5 mg IM) to induce estrus. Late diestral mares were allowed to return to estrus without treatment. When mares were detected in estrus and exhibited a dominant follicle greater than 30 - $35 \mathrm{~mm}$ on transrectal ultrasound, 1500 2000 international units (IU) of human chorionic gonadotropin (hCG, Chorulon, Intervet Schering Plough Animal Health Corp), depending on body weight, were administered intravenously to induce ovulation. Twentyfour hours later, mares were inseminated with 30 million progressively motile spermatozoa extended in commercial semen extender (INRA 96, IMV Technologies) in the uterine body.

Semen was collected from a single fertile Quarter Horse stallion using a Colorado-model artificial vagina. Immediately following collection, laboratory analysis of raw and extended semen was performed, including photometric measurement of sperm concentration (Densimeter; Animal Reproduction Systems) and measurement of motility and morphology via light microscopy (Figure 1). The fresh semen was extended with INRA 96 (IMV Technologies) up to a maximum volume of $10 \mathrm{~mL}$, with the goal of obtaining a final concentration of 25 million sperm $/ \mathrm{mL}$. Each insemination dose contained 30 million progressively motile spermatozoa and was protected from light until insemination. Seven mares housed onsite were inseminated with fresh extended semen, whereas semen was cooled for up to 8 hours in a commercial shipper (Equine Express II Cooled Semen Shipper; Exodus Breeders Corporation) prior to insemination of seven mares located in Raleigh.

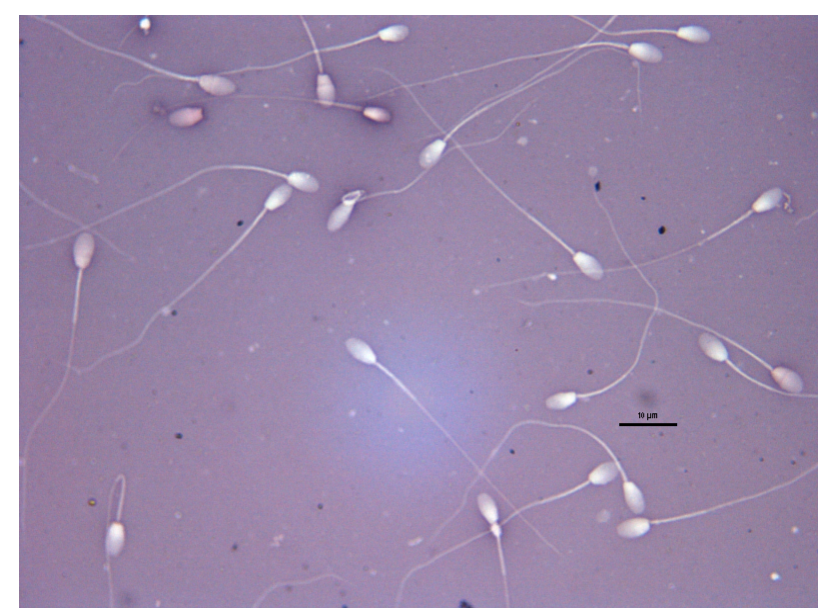

Figure 1. Equine semen sample stained with Eosin-Nigrosin Stain for evaluation of morphologic characteristics. 
Mares were restrained in stocks with their tails wrapped and tied to the side away from the vulva and perineum for artificial insemination. After removing feces from the rectum, the perineal area was washed with betadine scrub, rinsed with water, and dried with a paper towel to remove fecal contamination. Insemination of all mares was performed by a single individual. Wearing a sterile lubricated glove, an insemination pipette was passed through the vulvar lips into the vaginal vault. The insemination pipette was then guided through the external cervical os and cervix and advanced 1 to $2 \mathrm{~cm}$ into the uterine body before depositing the semen. Immediately following insemination, mares were given either phenylephrine (0.06 $\mathrm{mg} / \mathrm{kg}$ ) or $1 \mathrm{~mL}$ of saline via intravenous injection. On the following day, mares were examined via transrectal ultrasonography to detect ovulation. If ovulation had not occurred, mares were inseminated and treated a second time as described above. Mares that did not ovulate within 72 hours of the hCG administration or which had evidence of endometritis were excluded from this study. Pregnancy status was determined 14 days following ovulation via transrectal ultrasonographic examination to identify the presence of an embryonic vesicle (Figure 2). At this time, pregnant animals received Prostaglandin $F_{2 \alpha}$ (Lutalyse, Pfizer, $5 \mathrm{mg} \mathrm{IM}$ ) to terminate the pregnancy. A priori analysis using the program GPower 3.1 (G* Power, Düsseldorf, Germany) was performed prior to the onset of the study. Based on previous work with the stallion enrolled in this study and the results of Hawk and coworkers, power analysis indicated the need for 14 animals, examined over 2 cycles. At the conclusion of the study, data were statistically examined for differences between groups, differences between the first and second cycle in the study, differences between farms, and differences between mares inseminated once or twice. The statistical software program Statistix 8.1 (Statistix ${ }^{\circledR}$, Analytical Software Inc, Tallahassee FL) was utilized for all analyses and data were tested using Wilkoxon Rank Sum tests. Differences were considered significant at $P<$ 0.05 .

\section{RESULTS}

Out of a total of 28 possible cycles from 14 mares during the period of the study, 18 cycles qualified for inclusion in the analysis. Three cycles were excluded because the mares failed to ovulate within 72 hours of hCG administration, 4 cycles were excluded because mares ovulated unexpectedly before insemination, and 3 cycles were excluded due to ultrasonographic or cytologic evidence of endometritis. Of the 18 included cycles, the pregnancy rate was $44 \%$ (4/9) for phenylephrine treated cycles and 22\% (2/9) for the saline-treated cycles $(P=0.62$; Table 1). No differences were detected in pregnancy rates by location (3 pregnant mares in each location), cycle (4

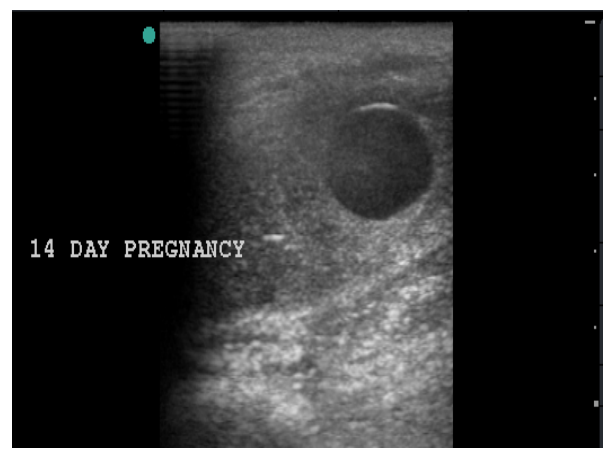

Figure 2. Ultrasonographic image of an equine embryonic vesicle 14 days post-ovulation.

Table 1. Pregnancy rates in mares after insemination with 30 million spermatozoa and treatment with phenylephrine or saline.

\begin{tabular}{ccccc}
\hline & Pregnant & Open & $\begin{array}{c}\text { Total } \\
\text { Estrous } \\
\text { Cycles }\end{array}$ & Pregnancy Rates \\
\hline $\mathrm{TX}^{\mathrm{a}}$ & 4 & 5 & 9 & $44 \%$ \\
$\mathrm{CT}^{\mathrm{b}}$ & 2 & 7 & 9 & $22 \%$ \\
\hline
\end{tabular}

${ }^{a}$ Phenylephrine $\mathrm{HCl}(0.06 \mathrm{mg} / \mathrm{kg})$ injected intravenously immediately after insemination; ${ }^{\mathrm{b}} 1 \mathrm{ml}$ of Saline injected intravenously immediately after insemination.

pregnancies were detected during the first cycle, 2 during the second), insemination number (3 pregnant mares were inseminated once, 3 were inseminated twice).

\section{DISCUSSION}

Altering contractility may be a viable means of improving pregnancy rates in mares when suboptimal doses of sperm or compromised sperm are used for insemination. This is supported by studies in rabbits and sheep, which demonstrate that phenylephrine and other uterotonic agents enhance uterine contractility, sperm numbers at the site of fertilization and fertilization rates [10-12]. These studies also demonstrated, however, that any positive effect on pregnancy rates is likely dose-specific. In mares, convincing evidence also exists to suggest that uterine contractility is an important component of sperm transport [13-17]. Artificial insemination alone has been shown to cause an initial increase in the myoelectrical activity of the uterus, resulting in transport of sperm through the female tract [18]. A second increase in myoelectrical activity, beginning 4 hours post breeding and lasting up to 12 hours, results from an inflammatory reaction to the semen and is associated with semen clearance from the reproductive tract [18]. Current research in mares is limited by the difficulty of objectively quantifying uterine contractility in response to insemination or drug administration. Most techniques described 
are invasive or not applicable to fertile breedings $[14,15,18]$. While less invasive, ultrasonographic evaluation of uterine contractility is subjective and may not be sufficiently sensitive to differentiate propulsive contractions from those that serve to evacuate the uterus. Consequently, the outcome selected for this and prior studies has been pregnancy rates in treated and untreated mares. While no statistical differences were detected in the current study, a doubling in pregnancy rates from $22 \%$ to $44 \%$ in phenylephrine-treated mares may be clinically important. Varner and coworkers demonstrated similar numerical improvements in pregnancy rates with PGE infused into the tip of the uterus [9], whereas intravenous and intramuscular oxytocin administration resulted in a numerical decrease in pregnancy rates compared to saline controls [8]. While higher pregnancy rates would be desired, a 44\% pregnancy rate approaches that of other methods described for low dose insemination $[4,6,9]$ and may be clinically useful, particularly given the ease of administration.

There were several limitations to the current study: the exclusion of 10 cycles from analysis reduced the statistical power of the study; further, the relatively high pregnancy rate found in the control group was unexpected based on previous work with this stallion. A larger sample population of mares and a smaller dose of motile sperm (5 million or fewer) may have resulted in statistical differences.

Altering the phenylephrine dose may enhance propulsive contractions and improve pregnancy rates in future studies. The current dose was selected based on its prior use in horses to induce contraction of the spleen in the treatment of nephrosplenic entrapment, however the uterine effects of this dose have not been evaluated. In rabbits, Hawk and coworkers demonstrated that $5 \mathrm{mg}$ of phenylephrine, but not a higher or lower dose, was effective at enhancing sperm delivery to the oviduct [10]. Further, the route of administration selected in this study may have affected the outcome. While phenylephrine is generally administered to horses intravenously to treat nephrosplenic entrapments, the drug was administered intramuscularly in the rabbit and ewe. Changing the route of administration may result in an alteration of the intensity or timing of uterine contractions to promote sperm-transport in the desired direction.

\section{CONCLUSION}

In conclusion, although this study failed to demonstrate a statistical improvement in pregnancy rates, the numerical increase may be clinically relevant. The results suggest that pregnancy rates after low dose insemination of mares might be improved by the use of uterotonic agents including phenylephrine. A sensitive and non-invasive technique is needed to measure uterine contractile patterns in response pharmacologic stimuli, to best determine the clinically appropriate dose.

\section{ACKNOWLEDGEMENTS}

The authors thank Jori Vasgaard for her invaluable technical and editorial assistance.

\section{REFERENCES}

[1] First, N.L., Short, R.E., Peters, J.B. and Stratman, F.W. (1968) Transport and loss of boar spermatozoa in reproductive tract of sow. Journal of Animal Science, 27, 1037-1040.

[2] Overstreet, J.W. and Cooper, G.W. (1978) Sperm transport in reproductive-tract of female rabbit .1. Rapid transit phase of transport. Biology of Reproduction, 19, 101114. http://dx.doi.org/10.1095/biolreprod19.1.101

[3] Bader. H. (1981) Sperm population in the genital-tract of the mare. Zuchthygiene-Reproduction in Domestic Animals, 16, 72-73.

[4] Lindsey, A.C., Bruemmer, J.E. and Squires, E.L. (2001) Low dose insemination of mares using non-sorted and sex-sorted sperm. Animal Reproduction Science, 68, 279289. http://dx.doi.org/10.1016/S0378-4320(01)00165-8

[5] Mari, G., Santi, A., Merlo, B., Iacono, E., Mislei, B. and Roberts, W. (2006) Pregnancy rates obtained with low numbers of frozen spermatozoa inseminated into the tip or the body of the uterus in mares. Animal Reproduction Science, 94, 378-380. http://dx.doi.org/10.1016/j.anireprosci.2006.04.029

[6] Brinsko, S.P., Rigby, S.L., Lindsey, A.C. Blanchard, T.L., Love, C.C. and Varner, D.D. (2003) Pregnancy rates in mares following hysteroscopic or transrectally-guided insemination with low sperm numbers at the utero-tubal papilla. Theriogenology, 59, 1001-1009. http://dx.doi.org/10.1016/S0093-691X(02)01123-8

[7] Morris, L.H.A., Hunter, R.H.F. and Allen, W.R. (2000) Hysteroscopic insemination of small numbers of spermatozoa at the uterotubal junction of preovulatory mares. Journal of Reproduction and Fertility, 118, 95-100.

[8] Rigby, S., Hill, J., Miller, C., Thompson, J., Varner, D. and Blanchard, T. (1999) Administration of oxytocin immediately after insemination does not improve pregnancy rates in mares bred by fertile or subfertile stallions. Theriogenology, 51, 1143-1150. http://dx.doi.org/10.1016/S0093-691X(99)80017-X

[9] Woods, J., Rigby, S., Brinsko, S., Stephens, R., Varner, D. and Blanchard, T. (2000) Effect of intrauterine treatment with prostaglandin E-2 prior to insemination of mares in the uterine horn or body. Theriogenology, 53, 1827-1836. http://dx.doi.org/10.1016/S0093-691X(00)00318-6

[10] Hawk, H.W., Cooper, B.S. and Conley, H.H. (1982) Increased numbers of sperm in the oviducts and improved fertilization rates in rabbits after administration of phenylephrine or ergonovine near the time of insemination. Journal of Animal Science, 55, 878-890. 
[11] Hawk, H.W. and Conley, H.H. (1985) Effect of prostaglandin-F2-alpha, phenylephrine and ergonovine on uterine contractions in the ewe. Journal of Animal Science, 60, 537-543.

[12] Hawk, H.W. and Cooper, B.S. (1984) Improvement by ergonovine of sperm transport, fertilization and pregnancy rates in ewes in natural or prostaglandin-induced estrus. Journal of Animal Science, 59, 754-763.

[13] Katila, T. (1999) Uterine contractility in nonpregnant mares. Pferdeheilkunde, 15, 574-578.

[14] Katila, T. (2001) Sperm-uterine interactions: A review. Animal Reproduction Science, 68, 267-272. http://dx.doi.org/10.1016/S0378-4320(01)00162-2

[15] Troedsson, M.H.T., Liu, I.K.M. and Crabo, B.G. (1998)
Sperm transport and survival in the mare: A review (vol 49, pg 905, 1998). Theriogenology, 50, 807-818.

[16] Campbell, M.L.H. and England, G.C.W. (2002) M-mode ultrasound imaging of the contractions of the equine uterus. Veterinary Record, 150, 575-577. http://dx.doi.org/10.1136/vr.150.18.575

[17] Campbell, M.L.H. and England, G.C.W. (2004) Effect of teasing, mechanical stimulation and the intrauterine infusion of saline on uterine contractions in mares. Veterinary Record, 155, 103. http://dx.doi.org/10.1136/vr.155.4.103

[18] Troedsson, M.H.T., Steiger, B.N., Ibrahim, N.M., King, V.L., Foster, D.N. and Crabo, B.G. (1995) Mechanism of sperm-induced endometritis in the mare. Biology of Reproduction, 52, 133-133.

\section{LIST OF ABBREVIATIONS}

mg: milligram

IM: intramuscular

IU: intrauterine

hCG: human chorionic gonadotropin

$\mathrm{mL}$ : milliliter 